\title{
¿Es la comunicación quisto-biliar, un factor de riesgo para el desarrollo de morbilidad postoperatoria en pacientes con hidatidosis hepática?*
}

\author{
Drs. CARLOS MANTEROLA D..$^{1,2}$, LUIS BUSTOS M. ${ }^{2}$, \\ MANUEL VIAL G ${ }^{1}$, JAVIER MORAGA C. ${ }^{1}$. GRUPO MINCIR. \\ Departamento de Cirugía, Universidad de La Frontera, Temuco, Chile. \\ 2 CIGES (Capacitación, Investigación y Gestión para la Salud Basada en Evidencia). Universidad de La Frontera, \\ Temuco, Chile.
}

\begin{abstract}
Intrabiliary rupture of hydatid cysts as a risk factor for postoperative complications

Background: Intrabiliary rupture is a complication of hepatic hydatid cysts. Aim: To determine if intrabiliary rupture is a risk factor for postoperative complications of hydatid cysts. Material and Methods: Prospective follow up of patients operated for hepatic hydatid cysts between 1996 and 2006. Patients were evaluated every six months during the first years and every 12 months thereafter. The presence of intrabiliary rupture was evaluated as a risk factor to develop complications during the follow up. Results: Ninety six patients with and 156 patients without intrabiliary rupture, $56 \%$ females, aged 42 years, were followed for a mean of 86.5 months. The overall incidence of complications was $17 \%$. The incidence in patients with and without intrabiliary rupture was 9,4 and $21,8 \%$ respectively $(\mathrm{p}=0.01)$. The bivariate analysis showed differences between patients with and without complications in leukocyte count, serum bilirubin, alkaline phosphatases, transaminases, cyst diameter, hospital stay and cyst complications. The relative risk for complications of intrabiliary rupture was 3,4 (95\% confidence intervals 2,6-4,2). Conclusions: The presence of intrabiliary rupture of a hepatic hydatid cyst is an independent risk factor for the development of complications in the postoperative period.
\end{abstract}

Key words: Intrabiliary rupture, hydatid cyst, complications.

\section{Resumen}

Introducción: Una de las complicaciones evolutivas de la hidatidosis hepática (HH) es la comunicación quisto-biliar (CQB). El objetivo de este estudio es determinar si la CQB es un factor de riesgo para el desarrollo de morbilidad postoperatoria en pacientes con HH. Material y Método: Estudio de cohorte prospectiva. Pacientes intervenidos quirúrgicamente por HH entre 1996 y 2006 en el Hospital Regional de Temuco, Chile, con seguimiento mínimo de 12 meses y controles clínicos en los meses 1, 6, 12, 24, 36, 48 y 60. La variable resultado fue "desarrollo de morbilidad postoperatoria", considerada de forma dicotómica. La variable de exposición fue presencia de CQB; analizada en forma dicotómica (CQB presente o ausente);

*Recibido el 5 de Diciembre de 2008 y aceptado para publicación el 17 de Enero de 2009.

Correspondencia: Dr. Carlos Manterola D.

Casilla 54-D, Temuco, Chile. Fax: 45-325761

E-mail: cmantero@ufro.cl 
y según el número de CQB ( $\sin \mathrm{CQB}$, con una CQB y con dos o más CQB). El tamaño de la muestra fue estimado en base a intervalo de confianza de $95 \%$, un poder de $80 \%$, relación pacientes con CQB: sin CQB de 1:1; proporción de morbilidad postoperatoria en pacientes con CQB de $30 \%$, y de $14 \%$ en pacientes sin CQB. Se utilizó estadística descriptiva, análisis bivariados y multivariados, con modelos de regresión logística ordinal; de este modo se calcularon riesgos relativos (RR) y sus respectivos intervalos de confianza del 95\% (IC). Resultados: Las cohortes están compuestas por 96 pacientes sin CQB $(38,1 \%)$ y 156 pacientes con CQB $(61,9 \%)$. Se caracterizan por una mediana de edad de 42 años; $56,4 \%$ de género femenino, una mediana de diámetro quístico y de seguimiento de $15 \mathrm{~cm}$ y 86,5 meses respectivamente. Se verificó un $17,1 \%$ de morbilidad. La morbilidad del grupo sin CQB fue de $9,4 \%$ y del grupo con CQB de $21,8 \%$ $(\mathrm{p}=0,011)$. Del análisis bivariado destacan diferencias entre grupos en las variables recuento de leucocitos, bilirrubina, fosfatasa alcalina, transaminasas, diámetro del quiste, estancia hospitalaria y existencia de complicación del quiste $(\mathrm{p}<0,01)$. Se verificó un RR de 3,4 (IC de 2,64, 4,18) para la comparación de subgrupos sin $\mathrm{CQB}$ o con dos o más CQB $(\mathrm{p}<0,001)$. Conclusión: La presencia de dos o más CQB constituye un factor de riesgo para el desarrollo de morbilidad postoperatoria en pacientes con $\mathrm{HH}$.

Palabras clave: Hidatidosis, hidatidosis hepática, quiste hidatídico hepático, morbilidad, factores de riesgo.

\section{Introducción}

Una de las complicaciones evolutivas más frecuentes de la hidatidosis hepática (HH) es la ruptura intrabiliar, que genera posteriormente una comunicación quisto-biliar $(\mathrm{CQB})^{1,2}$. Ésta, cuya prevalencia reportada varía entre $9 \%$ a $42 \%{ }^{2-10}$, puede favorecer el paso de vesículas hijas o trozos de la germinal a la vía biliar, produciendo colangiohidatidosis ${ }^{11} \mathrm{o}$ la infección del quiste, desarrollándose un absceso hepático secundario ${ }^{12}$; las situaciones antes descritas pueden incrementar el riesgo de complicaciones postoperatorias ${ }^{13}$.

Sin embargo, el diagnóstico preoperatorio de CQB es difícil y, con excepción de aquellas situaciones en las que se ha desarrollado una complicación evolutiva avanzada ${ }^{11,12}$, es difícil predecir la existencia de una CQB y el diagnóstico sólo se logra realizar durante la cirugía cuando se constata la salida de bilis al interior del quiste una vez drenado el contenido de éste ${ }^{14}$.

Por otra parte, existe evidencia tipo 4 que sugiere que existe asociación entre la presencia de CQB y el desarrollo de morbilidad postoperatoria, en pacientes intervenidos quirúrgicamente por $\mathrm{HH}^{3,4,8-10,13,15-17}$.

El objetivo de este estudio es determinar si la presencia de CQB es un factor de riesgo para el desarrollo de morbilidad postoperatoria en pacientes con $\mathrm{HH}$.

\section{Material y Método}

Diseño del estudio: Estudio de cohorte prospectiva.

Población en estudio: Pacientes intervenidos quirúrgicamente por $\mathrm{HH}$ en el período enero de 1996 a diciembre de 2006 en el Hospital Regional de Temuco, por el primer autor (CM); el tiempo de seguimiento mínimo es de 12 meses. Se excluyeron pacientes con antecedentes de cirugía previa por $\mathrm{HH}^{13}$, absceso hepático de origen hidatídico ${ }^{12}$, litiasis biliar y otras patologías hepáticas concomitantes. Se utilizó un muestreo no probabilístico de casos consecutivos.

Protocolo de estudio: Todos los pacientes fueron estudiados mediante pruebas diagnósticas generales, de función hepática, inmunodiagnóstico, radiología de tórax y ecotomografía abdominal. Después del alta hospitalaria, los pacientes fueron seguidos con controles clínicos estrictos a lo menos en los meses $1,6,12,24,36,48$ y 60 , oportunidad en la que se realizaba evaluación clínica, de laboratorio general, función hepática, inmunodiagnóstico y ecotomografía abdominal.

Variables en estudio: La variable resultado fue "desarrollo de morbilidad postoperatoria", medida al menos 12 meses después de la cirugía. Ésta fue considerada de forma dicotómica (es decir, presente o ausente). La variable de exposición fue presencia de CQB, la que se analizó desde dos perspectivas: la primera, en forma dicotómica (CQB presente o ausente); y la segunda, según el número de $\mathrm{CQB}$, categorizando la variable en $\sin \mathrm{CQB}$, con una $\mathrm{CQB}$ y con dos o más CQB. Las variables de control fueron: edad, género, diámetro del quiste, hematocrito, recuento total de leucocitos, bilirrubina total, fosfatasas alcalinas y transaminasas, tipo de cirugía realizada (categorizada en quistectomía subtotal ${ }^{18}$, periquistectomía total y resección hepática), existencia de complicaciones evolutivas del quiste (dicotomizadas en sí o no), estancia hospitalaria, necesidad de reintervención quirúrgica y mortalidad operatoria.

Tamaño de la muestra: Se estimó tomando en 
consideración los datos aportados por estudios pre$\operatorname{vios}^{3,4,6,15,19}$. Con un intervalo de confianza de $95 \%$, un poder de $80 \%$, una relación pacientes con CQB: sin CQB de 1:1, una proporción de complicaciones en pacientes con CQB de $30 \%$ y de $14 \%$ en pacientes sin CQB; se calculó una muestra mínima para la conducción del estudio de 94 sujetos con CQB y 94 sin CQB para la conducción del estudio.

Definiciones: Se definió como complicaciones evolutivas del quiste la existencia de tránsito hepatotorácico, rotura del quiste a cavidad abdominal, siembra peritoneal hidatídica y anafilaxia secundaria $^{20}$.

Plan de análisis: Los datos fueron analizados con el programa Stata $9.0 / \mathrm{SE}^{\circledR}$. Se utilizó estadística descriptiva, con cálculo de porcentajes, promedios y desviaciones estándar, medianas y valores extremos; posteriormente, se aplicaron análisis bivariados, utilizando prueba exacta de Fisher para variables categóricas, ANOVA y comparaciones múltiples de Scheffe y Kruskal-Wallis para variables continuas; $y$, finalmente, para estudiar fuerza de asociación, se aplicaron análisis multivariados con modelos de regresión logística ordinal en los que se seleccionaron las mejores variables que predecían el número de CQB, utilizando el procedimiento de selección de variables "backward stepwise", considerando probabilidad de entrada de 0,05 y de salida 0,40 . De este modo se calcularon riesgos relativos (RR) y sus respectivos intervalos de confianza del $95 \%$.

\section{Resultados}

Ingresaron al estudio un total de 261 pacientes intervenidos quirúrgicamente por $\mathrm{HH}$ en el período estudiado, de los cuales 9 no fueron considerados en el análisis final debido a pérdida de seguimiento (3 pacientes del grupo sin CQB y 6 con CQB); por ende, las cohortes en comparación están compuestas por 96 pacientes sin CQB $(38,1 \%)$ y 156 pacientes con CQB $(61,9 \%)$.

Los 252 pacientes que componen las cohortes en estudio se caracterizan, en general, por una mediana de edad de 42 años (15 a 84 años), 56,4\% de género femenino y tener un $33,7 \%$ de ellos dos o más lesiones quísticas con una mediana de diámetro de $15 \mathrm{~cm}(5$ a $30 \mathrm{~cm})$. Se registró una mediana de estancia hospitalaria de 5 días (2 a 40 días) y una mediana de seguimiento de 86,5 meses ( 6 a 160 meses). Se verificó un 17,1\% de morbilidad; un índice de reintervenciones de $5,2 \%$ y mortalidad de $0,8 \%$.

La morbilidad relacionada fue secundaria a: complicaciones pulmonares (21 casos; 8,3\%), infección del sitio operatorio ( 9 casos; $3,6 \%$ ), hernia incisional (5 casos; $2,0 \%$ ), fístula biliar (4 casos; 1,6\%), infección del tracto urinario y evisceración (2 casos de cada cual; $1,6 \%$ ). La morbilidad del grupo sin CQB fue de 9,4\% y del grupo con CQB, de $21,8 \%(\mathrm{p}=0,011)$.

Comparando las cohortes en relación a la presencia o ausencia de CQB, el análisis bivariado destaca las diferencias entre grupos de las variables recuento total de leucocitos, bilirrubina, fosfatasas alcalinas, transaminasas, diámetro del quiste, estancia hospitalaria y existencia de complicación del quiste $(\mathrm{p}<0,01)$ (Tablas 1,2 y 3$)$.

Del análisis bivariado de las cohortes en relación al segundo escenario ( $\sin \mathrm{CQB}$, con una $\mathrm{CQB}$ y con dos o más CQB), destacan las diferencias entre grupos de las mismas variables del escenario anterior $(\mathrm{p}<0,01)$ (Tablas 4, 5 y 6).

Al aplicar los modelos de regresión logística ordinal, se verificó que las únicas variables que se

Tabla 1. Variables continuas y su asociación con la existencia de CQB

\begin{tabular}{lccc}
\hline Variable & $\begin{array}{c}\text { Sin CQB } \\
(\mathbf{n}=\mathbf{9 6})\end{array}$ & $\begin{array}{c}\text { Con CQB } \\
(\mathbf{n}=\mathbf{1 5 6})\end{array}$ & $\mathbf{p}$ \\
\hline Edad (años) & $45,1 \pm 18,6$ & $45,1 \pm 16,5$ & 0,9819 \\
Hematocrito $\left(\mathrm{x} \mathrm{mm}^{3}\right)$ & $38,9 \pm 4,1$ & $38,0 \pm 5,4$ & 0,1271 \\
Leucocitos $(\mathrm{x} \mathrm{mm})$ & $8.208,0 \pm 3.054,8$ & $9.641,5 \pm 4.227,1$ & 0,0042 \\
Bilirrubina (mg/dL) & $0,8 \pm 0,8$ & $1,6 \pm 2,7$ & 0,0060 \\
F. alcalina (U/L) & $323,3 \pm 307,3$ & $569,8 \pm 525,3$ & 0,0001 \\
ASAT (U/L) & $34,7 \pm 43,6$ & $62,6 \pm 100,2$ & 0,0105 \\
ALAT (U/L) & $39,9 \pm 86,3$ & $66,4 \pm 93,1$ & 0,0251 \\
Diámetro (cm) & $12,1 \pm 4,9$ & $16,3 \pm 6,8$ & 0,0001 \\
Estancia hospitalaria (días) & $4,9 \pm 2,9$ & $7,2 \pm 5,6$ & 0,0003 \\
\hline
\end{tabular}


asociaron con la categorización del número de CQB (ninguna, una, dos o más), fueron la morbilidad, el diámetro del quiste y los valores de fosfatasas alcalinas; observándose que el desarrollo de morbilidad se asocia con aquellas personas que presentan dos o más CQB (Figura 1).

Tabla 2. Variables dicotómicas y su asociación con la existencia de CQB

\begin{tabular}{lccc}
\hline Variable & $\begin{array}{c}\text { Sin CQB } \\
(\mathbf{n}=\mathbf{9 6})\end{array}$ & $\begin{array}{c}\text { Con CQB } \\
(\mathbf{n}=\mathbf{1 5 6})\end{array}$ & $\mathbf{p}$ \\
\hline Género (\%) & & & 0,618 \\
Femenino & 58,3 & 55,1 & \\
Masculino & 41,7 & 44,9 & \\
Complicación del quiste (\%) & & 0,003 \\
No & 76,0 & 57,7 & \\
Sí & 24,0 & 42,3 & \\
Número quistes (\%) & & & 0,514 \\
Uno & 68,8 & 64,7 & \\
Dos o más & 31,3 & 35,3 & \\
Necesidad de reoperación (\%) & & 0,070 \\
No & 97,9 & 93,0 & \\
Sí & 2,1 & 7,0 & \\
Mortalidad (\%) & & & 0,382 \\
No & 100,0 & 98,7 & \\
Sí & 0,0 & 1,3 & \\
\hline
\end{tabular}

El modelo de regresión logística ordinal ajustado permitió verificar un RR de $1,49(0,83,2,14)$ para la comparación de los subgrupos sin CQB y con una CQB (p < 0,001); y, un RR de 3,41 $(2,64,4,18)$ para la comparación de los subgrupos sin CQB y con dos o más CQB $(\mathrm{p}<0,001)$.

Tabla 3. Variables categóricas y su asociación con la existencia de CQB

\begin{tabular}{lccc}
\hline Variable & $\begin{array}{c}\text { Sin CQB } \\
(\mathbf{n}=\mathbf{9 6})\end{array}$ & $\begin{array}{l}\text { Con CQB } \\
(\mathbf{n}=\mathbf{1 5 6})\end{array}$ & $\mathbf{p}$ \\
\hline Localización del quiste (\%) & & & 0,477 \\
Lóbulo derecho & 60,4 & 66,0 & \\
Lóbulo izquierdo & 21,9 & 14,7 & \\
Bilateral & 15,6 & 15,4 & \\
Centrohepático & 2,1 & 3,9 & \\
Cirugía realizada (\%) & & & 0,149 \\
Quistectomía subtotal & 75,0 & 73,1 & \\
Periquistectomía & 16,7 & 10,3 & \\
Lobectomía & 8,3 & 16,6 & \\
& & & 0,083 \\
Tratamiento cavidad residual (\%) & & \\
Sin tratamiento & 57,3 & 43,0 & \\
Capitonaje & 21,9 & 27,5 & \\
Epiploplastia & 20,8 & 29,5 & \\
\hline
\end{tabular}

Tabla 4. Variables continuas y asociación con número de CQB

\begin{tabular}{lcccc}
\hline Variable & $\begin{array}{c}\text { Sin CQB } \\
(\mathbf{n}=\mathbf{9 6})\end{array}$ & $\begin{array}{c}\text { Con 1 CQB } \\
(\mathbf{n}=\mathbf{9 5})\end{array}$ & $\begin{array}{c}\text { Con 2 o más CQB } \\
(\mathbf{n}=\mathbf{6 1})\end{array}$ & $\mathbf{p}$ \\
\hline Edad (años) & $45,1 \pm 18,7$ & $44,0 \pm 17,1$ & $46,9 \pm 15,6$ & 0,5806 \\
Hematocrito $\left.(\mathrm{x} \mathrm{mm})^{3}\right)$ & $38,9 \pm 4,1$ & $38,1 \pm 5,4$ & $37,8 \pm 5,3$ & 0,2372 \\
Leucocitos (x mm $)^{*}$ & $8.208,0 \pm 3.054,8$ & $9.197,4 \pm 3.804,2$ & $10.333,1 \pm 4.763,2$ & 0,0071 \\
Bilirrubina (mg/dL)* & $0,8 \pm 0,8$ & $1,5 \pm 2,4$ & $1,8 \pm 3,3$ & 0,0072 \\
F. alcalinas (U/L) & $323,3 \pm 307,3$ & $499,8 \pm 415,3$ & $678,7 \pm 650,1$ & 0,0013 \\
ASAT (U/L)\& & $34,7 \pm 43,6$ & $65,5 \pm 118,4$ & $57,9 \pm 62,8$ & 0,0013 \\
ALAT (U/L) & $39,9 \pm 86,3$ & $67,6 \pm 106,3$ & $64,6 \pm 68,6$ & 0,0002 \\
Diámetro (cm) & $12,1 \pm 4,9$ & $15,4 \pm 6,8$ & $17,5 \pm 6,6$ & 0,0001 \\
Estancia hospitalaria (días) & $4,9 \pm 2,9$ & $7,2 \pm 5,8$ & $7,2 \pm 5,1$ & 0,0001 \\
\hline
\end{tabular}

*Diferencias estadísticamente significativas entre el grupo sin CQB y el grupo con dos o más CQB.

"Diferencias estadísticamente significativas entre el grupo sin CQB y los otros dos grupos.

${ }^{\&}$ Diferencias estadísticamente significativas entre el grupo sin CQB y el grupo con dos o más CQB. 
Tabla 5. Variables dicotómicas y asociación con número de CQB

\begin{tabular}{|c|c|c|c|c|}
\hline Variable & $\begin{array}{l}\text { Sin } C Q B \\
(n=96)\end{array}$ & $\begin{array}{c}\text { Con } 1 \text { CQB } \\
(n=95)\end{array}$ & $\begin{array}{c}\text { Con } 2 \text { o más CQB } \\
(n=61)\end{array}$ & $\mathbf{p}$ \\
\hline Género (\%) & & & & 0,280 \\
\hline Femenino & 58,3 & 60,0 & 47,5 & \\
\hline Masculino & 41,7 & 40,0 & 52,5 & \\
\hline \multicolumn{3}{|c|}{ Complicación evolutiva del quiste $(\%)^{*}$} & & 0,003 \\
\hline No & 76,0 & 52,6 & 65,6 & \\
\hline Sí & 24,0 & 47,4 & 34,4 & \\
\hline Número quistes & & & & 0,565 \\
\hline Uno & 68,8 & 67,4 & 60,7 & \\
\hline Dos o más & 31,3 & 32,6 & 39,3 & \\
\hline Morbilidad (\%) & & & & 0,006 \\
\hline No & 90,6 & 83,2 & 70,5 & \\
\hline $\mathrm{Si}^{\prime}$ & 9,4 & 16,8 & 29,5 & \\
\hline Necesidad de re & & & & 0,143 \\
\hline No & 97,9 & 93,7 & 91,8 & \\
\hline $\mathrm{Si}^{\prime}$ & 2,1 & 6,3 & 8,2 & \\
\hline Mortalidad (\%) & & & & 0,199 \\
\hline No & 100,0 & 97,9 & 100,0 & \\
\hline Sí & 0,0 & 2,1 & 0,0 & \\
\hline
\end{tabular}

*Diferencias estadísticamente significativas entre el grupo sin CQB y los otros dos grupos.

Tabla 6. Variables categóricas y asociación con número de CQB

\begin{tabular}{lcccc}
\hline Variable & $\begin{array}{c}\text { Sin CQB } \\
(\mathbf{n}=\mathbf{9 6})\end{array}$ & $\begin{array}{c}\text { Con 1 CQB } \\
(\mathbf{n}=\mathbf{9 5})\end{array}$ & $\begin{array}{c}\text { Con 2 o más CQB } \\
(\mathbf{n}=\mathbf{6 1})\end{array}$ & $\mathbf{p}$ \\
\hline Localización del quiste (\%) & & & & 0,192 \\
$\quad$ Lóbulo derecho & 60,4 & 63,2 & 70,5 & \\
$\quad$ Lóbulo izquierdo & 21,9 & 20,0 & 6,6 & \\
$\quad$ Bilateral & 15,6 & 13,7 & 18,0 & \\
$\quad$ Centrohepático & 2,1 & 3,2 & 4,9 & 0,068 \\
Cirugía realizada (\%) & & & & \\
$\quad$ Quistectomía subtotal & 75,0 & 68,4 & 80,3 & \\
$\quad$ Periquistectomía & 16,7 & 13,7 & 4,9 & \\
$\quad$ Lobectomía & 8,3 & 17,9 & 14,8 & \\
Tratamiento cavidad residual (\%) & & & & \\
$\quad$ Sin tratamiento & 57,3 & 47,4 & 36,1 & \\
$\quad$ Capitonaje & 21,9 & 24,2 & 32,8 & \\
$\quad$ Epiploplastia & 20,8 & 28,4 & 31,1 & \\
\hline
\end{tabular}




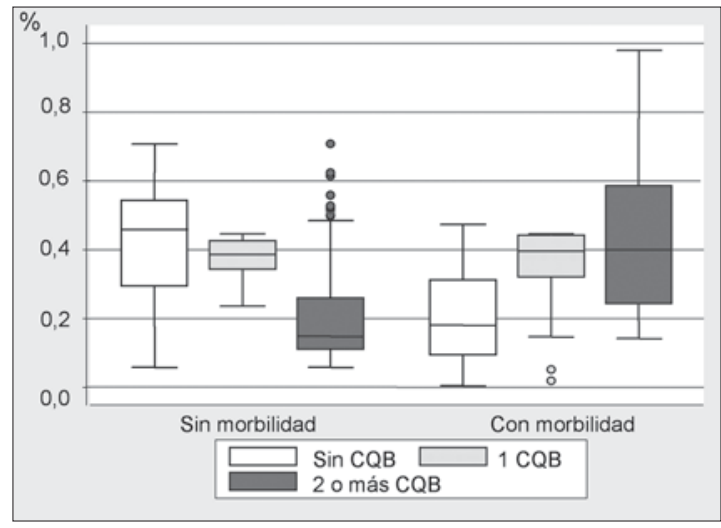

Figura 1. Probabilidades estimadas de desarrollar morbilidad postoperatoria según presencia y número de CQB después de aplicar el modelo de regresión logística.

\section{Discusión}

En relación al estudio de las CQB como factor asociado o factor de riesgo del desarrollo de morbilidad postoperatoria en la cirugía de la $\mathrm{HH}$ existen pocos estudios, la mayor parte de los cuales corresponden a series de casos de carácter retrospectivo, por lo que aportan un nivel de evidencia tipo $4^{21}$. Este es el primer estudio diseñado para valorar esta asociación y que se realiza utilizando un diseño apropiado para tal fin, es decir, un estudio de cohorte concurrente o prospectiva, que aporta un nivel de evidencia tipo $1 b^{21}$.

Aún así, somos conscientes que el estudio presenta algunas potenciales fuentes de sesgos que merecen ser comentadas. Una de ellas tiene que ver con la heterogeneidad de la población, hecho que se salvaguarda con el ajuste estadístico realizado al final del estudio. Otra, se relaciona con el tamaño de la muestra, el que se calculó con base a información previa que, como se comentó anteriormente, puede ser poco válida debido a la calidad metodológica de los estudios que la generaron; sin embargo, la valoración de la variable resultado enmascarada y realizada por los residentes del servicio de cirugía, la pérdida de seguimiento inferior a un $2 \%$ y un tiempo de seguimiento extenso, son herramientas que permiten incrementar la calidad de los datos.

Por otra parte, existe evidencia respecto de la asociación entre el desarrollo de CQB y morbilidad postoperatoria en estos pacientes. Es así como en un estudio comparativo, se observó un $54 \%$ de morbilidad en los pacientes con CQB y un $14 \%$ de morbilidad en aquellos sin $\mathrm{CQB}^{3}$; en un estudio de casos y controles anidado, se verificó que la CQB se asociaba a morbilidad postoperatoria en el análisis bivariado ${ }^{13}$; y, en distintas series de casos retrospectivas de pacientes con $\mathrm{CQB}$, se han reportado cifras de morbilidad entre $16,7 \%$ y $55 \%^{4,10,15-17}$.

Existen diferencias significativas en los análisis bivariados entre el grupo con y sin CQB, que dicen relación con algunas variables de control, las que se reiteran al recategorizar la variable de exposición de forma ordinal en tres categorías (sin CQB, con una CQB y con dos o más CQB). Este escenario de recategorizar la variable de exposición se originó al observar que un porcentaje importante de los pacientes del grupo en estudio tenían varias CQB y, al aplicar el modelo de regresión logística ordinal, se verificó una asociación estadísticamente significativa orientada al subgrupo de dos o más CQB $(p=0,001)$ con un riesgo relativo de 3,41. Lo anterior significa que la existencia de dos o más CQB incrementa en 3,4 veces el riesgo de desarrollar morbilidad postoperatoria en pacientes intervenidos quirúrgicamente por $\mathrm{HH}$.

Otro aspecto que vale la pena mencionar es la asociación entre el desarrollo de CQB con algunas variables que de alguna forma podrían explicar su formación. Al respecto, en una serie de casos de reciente publicación se identificó que las variables diámetro del quiste mayor de $10 \mathrm{~cm}$, quistes multivesiculares, infectados y localizados en el lóbulo izquierdo como variables asociadas al desarrollo de $\mathrm{CQB}^{22}$, situación que en nuestro estudio sólo se asemeja en relación a la variable "diámetro del quiste", debido a que la variable "infección del quiste" no pudo ser valorada al ser considerada como criterio de exclusión como posible confundente, al igual que el "antecedente de cirugía previa por HH", variable que en un estudio anterior demostró ser un factor de riesgo para el desarrollo de morbilidad postoperatoria en pacientes con $\mathrm{HH}^{13}$.

\section{Referencias}

1. Tacyildiz I, Aldemir M, Aban N, Keles C. Diagnosis and surgical treatment of intrabiliary ruptured hydatid disease of the liver. S Afr J Surg 2004; 42: 43-46.

2. Agarwal S, Sikora SS, Kumar A, Saxena R, Kapoor VK. Bile leaks following surgery for hepatic hydatid disease. Indian J Gastroenterol 2005; 24: 55-58.

3. Atli M, Kama NA, Yuksek YN, Doganay M, Gozalan $\mathrm{U}, \mathrm{Kologlu} \mathrm{M}$, et al. Intrabiliary rupture of a hepatic hydatid cyst: associated clinical factors and proper management. Arch Surg 2001; 136: 1249-1255.

4. Hamamci EO, Besim H, Sonisik M, Korkmaz A. Occult intrabiliary rupture of hydatid cysts in the liver. World 
J Surg 2005; 29: 224-226.

5. Kayaalp C, Bzeizi K, Demirbag AE. Biliary complications after hydatid liver surgery: incidence and risk factors. J. Gastrointest Surg 2002; 6: 706-712.

6. Paksoy M, Karahasanoglu T, Carkman S, Giray S, Senturk H, Ozcelik F, et al. Rupture of the hydatid disease of the liver into the biliary tracts. Dig Surg 1998; 15: 25-29.

7. Kornaros SE, Aboul-Nour TA. Frank intrabiliary rupture of hydatid hepatic cyst: diagnosis and treatment. J Am Coll Surg 1996; 183: 466-470.

8. Koksal N, Muftuoglu T, Gunerhan Y, Uzun MA, Kurt R. Management of intrabiliary ruptured hydatid disease of the liver. Hepatogastroenterol 2001; 48: 10941096.

9. Karydakis P, Pierrakakis S, Economou N, Ninos A, Raitsiou B, Bobotis E, et al. Surgical treatment of ruptures of hydatid cysts of the liver. J Chir 1994; 131: 363-370.

10. Atli M, Kama NA, Yuksek YN, Doganay M, Gozalan U, Kologlu M, et al. Intrabiliary rupture of a hepatic hydatid cyst: associated clinical factors and proper management. Arch Surg 2001; 136: 1249-1255.

11. Manterola C, Losada H, Carrasco R, Muñoz S, Bustos L, Vial M, y cols. Colangiohidatidosis. Una complicación evolutiva de la hidatidosis hepática. Bol Chil Parasitol 2001; 56: 10-15.

12. Manterola C, Barroso M, Vial M, Bustos L, Muñoz S, Losada $\mathrm{H}$, et al. Liver abscess of hydatid origin: clinical features and results of aggressive treatment. ANZ J Surg 2003; 73: 220-224.

13. Manterola C, Vial M, Pineda V, Sanhueza A, Barroso M. Factors associated with morbidity in liver hydatid surgery. ANZ J Surg 2005; 75: 889-892.

14. Koksal N, Muftuoglu T, Gunerhan Y, Uzun MA, Kurt
R. Management of intrabiliary ruptured hydatid disease of the liver. Hepatogastroenterol 2001; 48: 10941096.

15. Elbir O, Gundogdu H, Caglikulekci M, Kayaalp C, Atalay F, Savkilioglu M, et al. Surgical treatment of intrabiliary rupture of hydatid cysts of liver: comparison of choledochoduodenostomy with T-tube drainage. Dig Surg 2001; 18: 289-293.

16. Jabbour N, Shirazi SK, Genyk Y, Mateo R, Pak E, Cosenza DC, et al. Surgical management of complicated hydatid disease of the liver. Am Surg 2002; 68: 984988.

17. Wagholikar GD, Sikora SS, Kumar A, Saxena R, Kapoor VK. Surgical management of complicated hydatid cysts of the liver. Trop Gastroenterol 2002; 23: 35-37.

18. Manterola C, Molina E, Fernández O, Barroso M. Quistectomía subtotal. Una alternativa quirúrgica racional en el tratamiento de la hidatidosis hepática. Rev Chil Cir 1998; 50: 621-629.

19. Erzurumlu K, Dervisoglu A, Polat C, Senyurek G, Yetim I, Hokelek M. Intrabiliary rupture: an algorithm in the treatment of controversial complication of hepatic hydatidosis. World J Gastroenterol 2005; 11 : 2472-2476.

20. Manterola Delgado C, Barroso Vásquez M, Oberg Aravena C, Molina Villarroel E, Vial Gallardo M, Fernández Arancibia O. Alternativas quirúrgicas en el tratamiento de la hidatidosis hepática. Bol Chil Parasitol 1999; 54: 13-20.

21. http://www.cebm.net/index.aspx?o=1025. Accesado el 29 de Julio de 2007.

22. Al-Bahrani AZ, Al-Maiyah M, Ammori BJ, Al-Bahrani ZR. Factors predictive of frank intrabiliary rupture in patients with hepatic hydatid cysts. Hepatogastroenterol 2007; 54: 214-217. 